\title{
Perpectives and challenges of the triennium 2010-2012 for the postgraduate programs of the area 21 by CAPES
}

\author{
Perspectivas e desafios do triênio 2010-2012 para a pós-graduação na área 21 \\ da CAPES
}

In recent years, Brazil had one of the fastest rates of growth in scientific production and currently holds the $13^{\text {th }}$ position in the international ranking in the number of published articles (PNPG, 2011). In addition, the human resources have experienced a significant increase. This growth is determined by many factors, which involve from increment of resources to factors related to the national policies of induction. In this context, it is unequivocal the role of CAPES (Coordenação de Aperfeiçoamento de Pessoal de Nível Superior, Brasília, DF, Brazil) in the expansion and consolidation of the stricto sensu postgraduate system. The assessment system has been used as a tool for the community in order to search for academic excellence. Its results have been used for the formulation of policies for the postgraduate area as well as for the dimensioning of the actions of financial supports, such as grants, scholarships, fellowships, etc.

The act of assessing is not an easy task, and the intricate complexity of the factors considered by the assessment system proposed by CAPES (i.e., laboratories, libraries, employment status, intellectual production, social inclusion of the programme etc) makes this task even more difficult. Over the years and with a better understanding of these factors, many postgraduate programs have established their own strategies in order to align their activities with the evaluation parameters and these programs were able to achieve better scores. This fact has occurred especially with regards to the intellectual production, which has greater weight and is quite decisive in the assignment of the score. However, this process conferred to the postgraduate evaluation a negative aspect when it led some postgraduate programs to leave their characteristics and vocations behind de scenes, making the assessment one of their main goals. This fact diverts the purpose of inducing a natural growth in these postgraduate areas. An example of this fact is the deception used by some programs to insert highly productive researchers from other areas of knowledge that have little or no relationship to the area or research lines of the postgraduate program. If, on one hand, this strategy had shown to be positive in quantitative aspects and allowed the postgraduate program to obtain a higher score on the evaluation system, on the other hand, it produced a series of side effects. One of these side effects occurred by the incorporation of a considerable number of journals with low identity with the field of knowledge of the area, which led to the doubling of the median value of the main indexer (Journal Citation Reports-JCR) in the interstice of a triennium evaluation.

The increase of the JCR median cannot be exclusively attributed to this factor, because the need of the academics to search for more funding (such as grants, scholarships etc.) has led our researchers to publish in journals with better indexing (i.e., with higher impact factors). This has generally occurred in all areas of knowledge. However, the basic and experimental sciences journals have notoriously better indexes (which reflect the magnitude of the area) and started to occupy the upper strata of the Qualis (A1 and A2). Hence, journals that have a more applied scope, as well as the ones that have scope in the social and humanities sciences moved to the lower strata of the Qualis. In order to the area can be considered as "productive", it is not enough that their researchers publish in journals that are well indexed (generally of greatest impact factors), but that such publications keep close relationship with their 
working area and demonstrate relevance to the area 21. By following the logic of "productivity", it may lose the epistemological identity, which should guide researchers and programs of the area 21.

One of the challenges in the area 21 in the triennium 2010-2012 consists in the epistemological rescue of the area. Some strategies can be proposed to minimize these challenges in the medium and long term. A first possibility consists in a review of the evaluation criteria, allowing the consent to value academics that has a linkage of their intellectual production with areas connected to the lines and research projects of their respective programs. The second possibility is to give credit to specific journals of the area of knowledge of the postgraduate programs of the area 21. In addition, it must be considered the consolidated indexing databases (such as JCR, SJR, Medline, Scielo, Lilacs and others), which are widely used in the evaluations conducted by the other areas. Thus, the Qualis can reflect which of the journals in the area 21 belong to good indexing databases, considering moreover their identities with the subareas of the area 21 (Physical Therapy, Occupational Therapy, Speed Pathology and Physical Education).

To preserve the identity of the journals, the exclusion of articles that do not retain direct relationship with the area 21 must be embraced in the evaluation process. In fact, other areas of knowledge that are also evaluated by CAPES adopt this policy. Isolated publication can unambiguously qualify several researchers, however, it does not maintain the internal coherence of the postgraduate programs. It should be emphasized that the intellectual output evaluation aims to analyze its consistency with regards to the proposal and the goals of the postgraduate programs, and not exclusively to the judgment of the "quality" of their academic staff. Therefore, the exclusion of items that are not relevant to the area can help to reduce the distortions that end up increasing the median of the Qualis, without the area has defensive mechanisms of the identity of their field of expertise. These factors seem to aggravate even more the diversity found among the four sub-areas of area 21.

It is not only enough that the policies implemented by CAPES, specifically by the area 21 coordinators, to seek mechanisms to rescue the identity of its subareas; but also it is necessary for specific journals of the subareas (Physical Education, Physical Therapy, Speech Pathology and Occupational Therapy) to obtain inclusion in major indexing databases and could serve as vehicles recognized by national and international community. Accordingly, many journals have made great efforts and have received good results. The Revista Brasileira de Fisioterapia (RBF) is a clear example of this achievement. The current indexes of RBF indicate that it is possible that the area concentrates around causes that can consolidate the field. The change of quantity for quality of academic publishing vehicles is one of the main ways to succeed on this task. The Physical Therapy subarea has performed this task incontestably and serves as an example for the other 21 subareas, who are still in a consolidation phase of their journals, which are essential for the development of the area. The development of the area depends on the creation of new courses of master's and doctoral degrees. In recent years, the growth of the area 21 was expressive, but is still centered in the more developed regions of the country. The opening of courses in the North, Northeast and Midwest still a challenge to be overcome, with more incisive strategies, directed to the setting academics in regions mentioned above.

All subareas that comprise the area 21 are considered highly professional, which leads to the need to put on the agenda the professional master, by better defining the scope and evaluation criteria, making them more compatible with the desired capacitating and production. Currently, the area 21 has a single professional master's course in the Speech Pathology sub-area, demonstrating, therefore, the need for expansion. Other points to be discussed in depth in this triennium regards to the possibility of deploying a system for qualification assessment of books, which can be operated by researchers and by the programs themselves on an ongoing basis. The evaluation parameters of social inclusion, which would allow a better discrimination of the programs, require more objective assessment tools for its determination. Finally, this triennium presents some challenges to be overcome, for which we are counting with the collaboration of all academic staff linked to the postgraduate programs of the area 21.

André L. F. Rodacki

Coordinator of the area 21

Maria C. Martinelli

Adjunct Coordinator of the area 21

Rinaldo R. J. Guirro

Collaborator Coordinator of the area 21 